\title{
Quantitative assessment of myocardial extracellular volume fraction in non-ischemic dilated cardiomyopathy and its relation to systolic dysfunction
}

\author{
Aamir Ali ${ }^{*}$, Ankur Gulati ${ }^{1}$, Tevfik F Ismail ${ }^{1}$, Kaushiga Krishnathansan ${ }^{1}$, Nizar Ismail ${ }^{1}$, Evangelia Nyktari ${ }^{1}$, \\ Navtej Chahal', Claire E Raphael', Rick Wage', Taigang He', Peter D Gatehouse', David Firmin ${ }^{1}$, Peter Kellman², \\ Dudley Pennell', Andrew E Arai', Sanjay K Prasad ${ }^{1}$
}

From 16th Annual SCMR Scientific Sessions

San Francisco, CA, USA. 31 January - 3 February 2013

\section{Background}

Interstitial myocardial fibrosis is a histological hallmark of non-ischemic dilated cardiomyopathy (DCM), and may play an important role in adverse remodelling and progressive systolic dysfunction. T1-mapping enables noninvasive assessment of diffuse fibrosis by quantification of myocardial extracellular volume fraction (ECV). We hypothesized that CMR would identify a raised ECV in DCM which would correlate with the degree of systolic dysfunction.

\section{Methods}

Consecutive DCM patients referred for CMR and age/ sex-matched healthy controls were prospectively enrolled. Exclusion criteria included a history of recent myocarditis, ischemic heart disease, diabetes, severe hypertension and primary valvular disease. All subjects underwent CMR (1.5T, Siemens Avanto) according to a standardized protocol which included T1-mapping and late gadolinium enhancement (LGE) imaging. Mid-ventricular short-axis T1-maps were acquired using a Modified Look-Locker Inversion recovery sequence prior to contrast and 20 minutes after gadolinium administration (Gadovist $0.1 \mathrm{mmol} / \mathrm{kg}$ ). The pre- and post-contrast T1-maps were co-registered and used with the patient's hematocrit to generate an ECV map.

\section{Results}

In total, 85 patients (58 male, mean age $51.5 y$ rs, mean left ventricular ejection fraction [LVEF] 42\%) and 35 controls (22 male, mean age 46yrs, mean LVEF 68\%) were studied. Baseline clinical and CMR characteristics for the cohort are summarized in Table 1. Mid-wall LGE was present in $14(16.5 \%)$ DCM patients. In one patient, LGE was observed in the same short-axis slice as the T1-map and this study was therefore excluded from analysis. Patients with DCM had significantly higher ECV compared to controls $(28.8 \pm 3.9 \%$ vs. $25.5 \pm 3.2 \%, \mathrm{p}<0.0001)$. A significant negative correlation was observed between ECV and

\section{Table 1 Baseline clinical and CMR characteristics}

\begin{tabular}{cccc}
\hline Characteristic & Disease $(\mathbf{n}=\mathbf{8 5})$ & Control $(\mathbf{n}=\mathbf{3 5})$ & $\boldsymbol{p}$-value \\
\hline Age (years) & 52 & 46 & 0.067 \\
Male & 58 & 22 & 0.629 \\
Heart rate $(\mathbf{b p m})$ & 73 & 63 & $<0.0001$ \\
Systolic BP $(\mathbf{m m H g})$ & 122 & 120 & 0.698 \\
Diastolic BP $(\mathbf{m m H g})$ & 74 & 76 & 0.559 \\
LV-EDVi $(\mathbf{m L})$ & 133 & 81 & $<0.0001$ \\
LV-ESVi $(\mathbf{m L})$ & 80 & 26 & $<0.0001$ \\
LVEF & 41 & 68 & $<0.0001$ \\
LVMI (g/m $\left.\mathbf{m}^{\mathbf{2}}\right)$ & 82 & 57 & $<0.0001$ \\
\hline
\end{tabular}

Beats per minute (bpm); blood pressure (BP); indexed left ventricular enddiastolic colume (LV-EDVi); indexed left ventricular end-systolic volume (LV-ESVi); left ventricular ejection fraction (LVEF); indexed left ventricular mass (LVMI)

${ }^{1}$ CMR Unit, Royal Brompton Hospital, London, UK

Full list of author information is available at the end of the article

(c) 2013 Ali et al; licensee BioMed Central Ltd. This is an Open Access article distributed under the terms of the Creative Commons Attribution License (http://creativecommons.org/licenses/by/2.0), which permits unrestricted use, distribution, and reproduction in any medium, provided the original work is properly cited. 


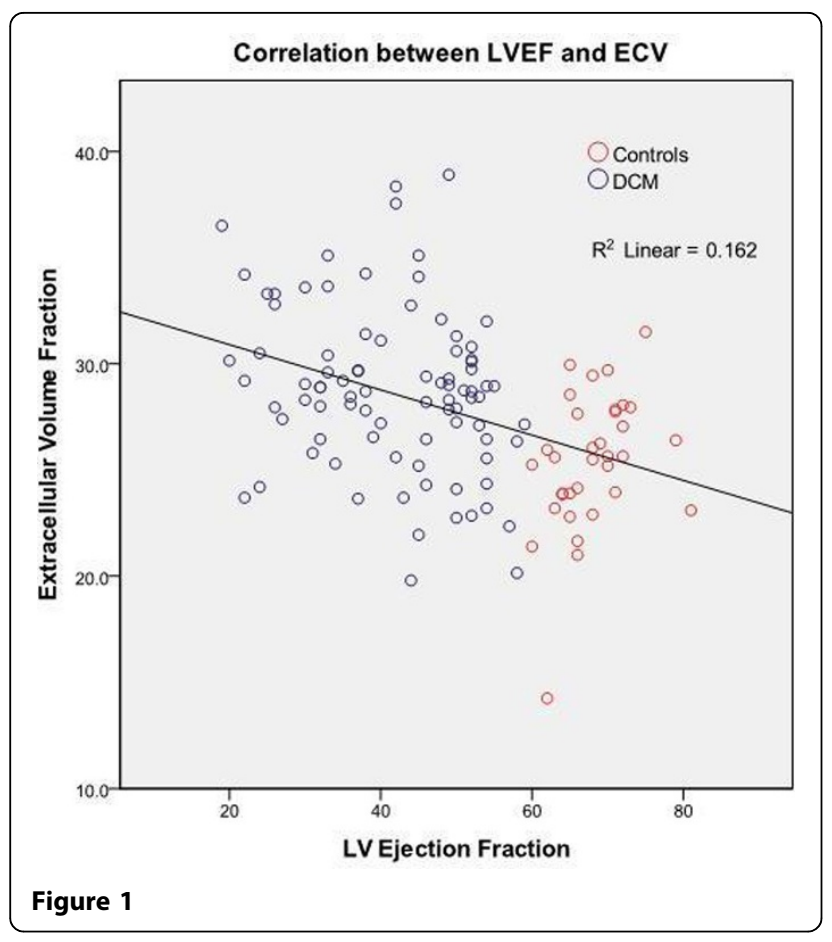

LVEF $(\mathrm{r}=-0.54, \mathrm{p}<0.0001)$. Univariate linear regression analysis revealed that indexed left ventricular end diastolic volume, heart rate, gender and LVEF were significantly associated with ECV. On multivariate analysis, only LVEF $(B=-0.12,95 \% C I-0.17$ to $-0.06, p<0.0001)$ and gender $(B=-3.2,95 \% \mathrm{CI}-4.6$ to $-1.9, \mathrm{p}<0.0001)$ were independent determinants of ECV.

\section{Conclusions}

ECV is expanded in DCM in proportion to the degree of LV systolic dysfunction. An increased ECV in sections of the heart without clinically obvious LGE suggests the presence of low level myocardial fibrosis or possibly myocardial edema. This technique offers potential for the evaluation of interstitial fibrosis in DCM, although important challenges include the substantial overlap of ECV values between patients and normals. Further work should aim to corroborate these findings with histological validation.

\section{Funding}

This project was supported by the NIHR Cardiovascular Biomedical Research Unit of Royal Brompton and Harefield NHS Foundation Trust, the British Heart Foundation, and CORDA (research charity).

Dr Peter Kellman and Dr Andrew Arai are funded by the National Heart, Lung and Blood Institute, NIH, Bethesda, MD, USA.

\section{Author details}

${ }^{1}$ CMR Unit, Royal Brompton Hospital, London, UK. ${ }^{2} \mathrm{NHLBI}$, National Institutes of Health, Bethesda, MD, USA.

Published: 30 January 2013

doi:10.1186/1532-429X-15-S1-019

Cite this article as: Ali et al:: Quantitative assessment of myocardial extracellular volume fraction in non-ischemic dilated cardiomyopathy and its relation to systolic dysfunction. Journal of Cardiovascular Magnetic Resonance 2013 15(Suppl 1):019.
Submit your next manuscript to BioMed Central and take full advantage of:

- Convenient online submission

- Thorough peer review

- No space constraints or color figure charges

- Immediate publication on acceptance

- Inclusion in PubMed, CAS, Scopus and Google Scholar

- Research which is freely available for redistribution

Submit your manuscript at www.biomedcentral.com/submit 